\title{
Correlation Between Role of Preoperative Computerized Tomography and Intraoperative Findings in Revision Myringoplasty \\ ${ }^{1}$ Mohammed Abd Elmoneim Ahmed, ${ }^{2}$ Abd Ellah Nazeer Yassin, \\ ${ }^{1}$ Yahia Mohammed Dawood, ${ }^{1}$ Ahmed Ragab Abd Elwahab \\ ${ }^{1}$ Department of Otorhinolaryngology, ${ }^{2}$ Department of Diagnostic Radiology, \\ Faculty of Medicine, Al-Azhar University \\ Corresponding author: Ahmed Ragab Abd Elwahab, Mobile: 01060123441, email: ragaboly90@ gmail.com
}

\begin{abstract}
Background: The goals of tympanic membrane repair in tubotympanic chronic suppurative otitis media are to close the perforation, remove the disease, and improve hearing of the patient. Myringoplasty is an operation used to repair a perforation of the tympanic membrane and this improves the patient hearing and decreases susceptibility of infection. Objective: To correlate the radiological findings of preoperative CT with intra-operative findings in revision myringoplasty.

Patients and Methods: This study included 30 patients suffering from safe type of chronic suppurative otitis media with central tympanic membrane perforations. 23 patients were subjected to underlay revision myringoplasty and 7 patients were subjected to cortical mastoidectomy based on the preoperative CT findings. Diagnosis of the disease was established by clinical, otoscopic, microscopic and endoscopic examination. All patients were studied prospectively. Preoperative history taking, ENT examination, general examination, and assessment of hearing were performed for all cases. All patients complained of history of ear discharge and deterioration of hearing. Surgery was done; operative details were recorded and postoperative follow up was done.

Results: This study showed that CT scan temporal bone is $100 \%$ accurate in assessment of ossicular chain, aditus ad antrum patency and mastoid pneumatization. While, CT accuracy in assessment of middle ear spaces, middle ear mucosa and fibrous adhesions is $76.7 \%$.
\end{abstract}

Conclusion: Comparing these results with those of other researches using similar techniques, revealed that they are almost similar regarding the accuracy of CT scanning and success rate.

Keywords: Chronic otitis media, tympanic membrane, chronic suppurative otitis media.

\section{INTRODUCTION}

The prevalence of chronic otitis media $(\mathrm{COM})$ is estimated at $0.5-30 \%$ in various populations, with approximately 120 million patients with COM worldwide. The primary treatment for $\mathrm{COM}$ is by surgery to eliminate the disease, prevent recurrence and maintain or improve hearing ${ }^{(\mathbf{1})}$. The objective of repairing the TMS in chronic tubular otitis media (CSOM) is to close the hole, remove the disease, and improve patient hearing ${ }^{(2)}$.

The term myringoplasty was firstly used in 1953 by Horst Wullstein when reporting his reconstruction technique of the tympano-ossicular system in cases of chronic otitis media. Regarding the technique, the targets of surgery are, the eradication of the pathological condition, resulting dry middle ear and restoration of the mechanisms that transmit sounds ${ }^{(3)}$.

Myringoplasty is a surgical method, to eradicate middle ear infection and improve its function. Type 1 tympanoplasty is the commonest otological procedure performed in any otological clinics for patients with COM. Different graft materials have been tried for reconstruction of the defect in the tympanic membrane with temporalis fascia being the most common autograft used ${ }^{(4)}$.

A main part of myringoplasty is repair of perforated TM which results mainly from COM.
Other etiologies include traumatic or neoplastic defects of the tympanic membrane ${ }^{(5)}$.

Various factors have been implicated to affect the results of myringoplasty; ventilation of middle ear is an essential predictor of the functional results following middle ear reconstruction. It is a complex and dynamic process depending on a number of factors including: the functional status of the Eustachian tube (ET), the degree of pneumatization of mastoid air cells, and the condition of middle ear mucosa. The role of mastoid pneumatization in the middle ear aeration is not exactly known. However, it forms an air reservoir and acts as a surge tank to minimize pressure fluctuation ${ }^{(6)}$.

Possible complications of myringoplasty include failure of the graft to heal, causing recurrent eardrum perforation, narrowing (stenosis) of the ear canal, scarring or adhesions in the middle ear, perilymph fistula, hearing loss, erosion or extrusion of the prosthesis, dislocation of the prosthesis and facial nerve injury. Other problems such as recurrence of cholesteatoma, may or may not result from the surgery ${ }^{(7)}$.

Failed tympanoplastic reconstruction still remains problematic. A number of causes have been recognized, including infection, poor wound healing, ET dysfunction, retained aural disease and poor surgical technique. Besides that, other studies consider material used for the graft, technique of the 
graft position and size of tympanic perforation predictive factors ${ }^{(8)}$.

CT scan added a new dimension to the study of middle ear disease; middle ear masses and associated complications were visualized for the first time ${ }^{(9)}$. Significant advances have occurred in the imaging of the ear structures with the development of a thin CT high-precision section. The bony portions of the temporal bone are depicted with approximately the same resolutions with polytomography, but CT added a whole new dimension for evaluation of the ear by allowing visualization of the soft tissue components within and adjacent to the temporal bone (10).

The temporal bone is usually imaged in two planes, most frequently axial and coronal. CT is most commonly used for the initial radiological evaluation of suspected developmental abnormalities, inflammatory processes, traumatic and destructive lesions ${ }^{(11)}$. CT scan is very effective in assessing the aditus occlusion and remaining tissue in the eardrum region ${ }^{(12)}$.

\section{AIM OF THE WORK}

The aim of this study is to correlate the radiological findings of preoperative $\mathrm{CT}$ with intraoperative findings in Revision Myringoplasty.

\section{PATIENTS AND METHODS}

This prospective study was carried out on 30 patients with CSOM with previous failed myringoplasty, 17 females (56.7\%) and 13 males (43.3\%), presented to outpatient clinic of Hearing and Speech Institute.

The age ranged from 20 to 50 years with mean age 33.80. All patients had previous history of myringoplasty without mastoidectomy.

Ethical approval was obtained from institutional board of Al-Azhar University and written consent was taken from every patient.

\section{All patients were subjected to:}

Preoperative assessment: Detailed history of the disease (onset, course, duration, frequency of exacerbations, and development of any complications and history of previous surgery to the ear). Otoscopic and microscopic examination of the ear. Examination of nose and throat. Nasopharyngeal examination by rigid 30 degree nasal endoscope. Audiological assessment: Tuning fork tests. Pure tone audiometry. Tympanometry. CT scanning of temporal bone axial and coronal cuts with $2 \mathrm{~mm}$ thickness and $2 \mathrm{~mm}$ interval.

All patients in this study must have had following criteria: History of previous myringoplasty without mastoidectomy. Dry perforation in pars tensa (any size and any shape). Patients with ear discharge were initially treated conservatively and were included in the study when the ear was dry for at least
6 weeks. Intact ossicular chain. No cartilage graft in the previous myringoplasty.

Patients with closed aditus ad antrum or nonpneumatized mastoid air cells which were confirmed by CT scan preoperatively, where the decision for revision myringoplasty with cortical mastoidectomy was taken. Systemic examination was done to assess fitness of the patient for operation and to exclude any general disease contraindicating surgery.

Preparations for operation:- Laboratory investigation. Complete blood picture, bleeding and clotting times, random blood sugar, urea and creatinine levels and SGOT and SGPT levels. ECG. Plain x-ray chest. The axial and coronal CT scanning of temporal bone without contrast with 2 mm slice thickness and $2 \mathbf{~ m m}$ intervals were taken preoperatively for every patient in this study for assessment of the tympanomastoid pneumatization, condition of aditus, ossicular chain and other middle ear spaces.

Operative procedure: Cases were operated upon through the period from October 2017 to July 2018. 23 patients were subjected to revision myringoplasty and 7 patients were subjected to revision myringoplasty with cortical mastoidectomy based on preoperative CT scan findings. Tragal cartilage graft was taken from all cases due to the ability of cartilage to resist infection, being easily available at the site of operation and causing minimum lateralization.

Surgical technique: General anesthesia: All patients were operated under general anesthesia with hypotensive technique. The ear was prepared with betadine solution exteriorly. The post auricular skin infiltrated with 3-5 ml saline -adrenaline $(1 / 200,000)$ for vasoconstriction. Curving incision from just above the pinna round to the mastoid tip, about $1 \mathrm{~cm}$ posterior to the sulcus was done. The incision was deepened to the plane between the deep fascia and temporalis fascia. The incision was deepened to the periosteum, which was incised and stripped by a periosteal elevator to expose the bony cortex. The pinna was held forwards and separated from the temporalis to expose the deep surface of the posterior metal wall, which was incised with elevation of the pinna by retractors. The flaps were held back using self retaining retractors. Trimming of the edge of the perforation. Dissection of the posterior meatal wall from 6 to 12 o'clock and elevation of the annulus with pushing of the meatal wall and drum remnant anteriorly to expose middle ear. Cortical mastoidectomy was done to 15 cases by using a large cutting burr 5-6 $\mathrm{mm}$ in diameter, the bone above and behind the external auditory meatus was gradually excavated to open the antrum. The bony posterior metal wall was thinned until short process of the incus was identified, then by curved needle; the soft tissue in the region of aditus ad antrum was removed and 
dissected until saline pass to the middle ear. Saline was injected in the antrum and its passage through the aditus to middle ear was noticed. Tragal cartilage graft was taken after injection of 1-3 ml saline adrenaline $(1 / 200,000)$ and dissection of the skin from the perichondrium at the inner side Then, making a cut at the site of incision and dissection of the other site of the cartilage.And finally, excision of a suitable piece of cartilage according to size of perforation and dissection of perichondrium from one side. Assessment of ossicular chain mobility for exclusion of any ossicular erosion or discontinuity was done for every case. Assessment of Eustachian tube patency was done by putting a finder into the Eustachian orifice to exclude any obstruction. The tragal cartilage graft was introduced under the handle of malleus and the perichondrium was contact with bony posterior metal wall. The tympano-meatal flap was repositioned. Gelfoam was applied to middle ear. Post auricular incision was closed in layers with interrupted sutures. Tragal incision was closed by one stitich and the external auditory canal was packed by gelfoam and sufratol and pressure bandage was applied.

All the intraoperative findings had been fulfilled into the operative sheet of ear surgeries performed by our institute and correlated with CT scan findings.

Follow up: Patients were hospitalized for one day, received systemic antibiotics, analgesics and nasal decongestant drops. A list of instructions was given and explained to the patients which included: avoiding water entry into the ear, discouraging nose blowing and activities such as sneezing with the mouth closed. Sutures and pack in the external canal were removed at the end of the week. Otoscopic and microscopic examination of the ear was done every week for 2 months.

\section{Statistical Analysis}

Data were collected, revised, coded and entered to the Statistical Package for Social Science (IBM SPSS) version 24 and the following were done:

Qualitative data were presented as number and percentages. (There is no quantitative data).

The confidence interval was set to $95 \%$ and the margin of error accepted was set to $5 \%$. So, the pvalue was considered significant as the following: $\mathrm{P}>$ 0.05: Non significant. P < 0.05: Significant. P < 0.01: Highly significant.

\section{RESULTS}

Table (1): Pre-operative CT findings

\begin{tabular}{|l|l|c|c|}
\hline \multicolumn{2}{|c|}{ Middle ear space } & Count & \% \\
\hline \multirow{2}{*}{ Ossicular chain } & clear middle ear space & 30 & $100.0 \%$ \\
\hline \multirow{2}{*}{ Aditus ad antrum } & intact ossicular chain & 30 & $100.0 \%$ \\
\cline { 2 - 4 } & Disrupted & 0 & $0.0 \%$ \\
\hline \multirow{2}{*}{$\begin{array}{l}\text { Mastoid } \\
\text { pneumatization }\end{array}$} & patent aditus & 25 & $83.3 \%$ \\
\hline blocked aditus & 5 & $16.7 \%$ \\
\hline Combined pathology & well pneumatized mastoid & 26 & $86.7 \%$ \\
\cline { 2 - 4 } Sclerotic patches & sclerosed mastoid & 4 & $13.3 \%$ \\
& blocked aditus and sclerosed mastoid & 2 & $6.7 \%$ \\
\cline { 2 - 4 } & no sclerotic patches & 27 & $90.0 \%$ \\
\cline { 2 - 4 } & Tympanosclerosis & 3 & $10.0 \%$ \\
\hline
\end{tabular}

Table (2): Surgical findings.

\begin{tabular}{|l|l|c|c|}
\hline \multirow{3}{*}{ Middle ear space } & clear middle ear & Count & \% \\
\cline { 2 - 4 } & polypoid middle ear mucosa & 23 & $76.7 \%$ \\
\cline { 2 - 4 } & middle ear adhesions & 3 & $10.0 \%$ \\
\hline \multirow{2}{*}{ Ossicular chain } & intact ossicular chain & 4 & $13.3 \%$ \\
\cline { 2 - 4 } & Disrupted & 30 & $100.0 \%$ \\
\hline \multirow{2}{*}{ Aditus ad antrum } & patent aditus & 0 & $0.0 \%$ \\
\cline { 2 - 4 } & blocked aditus & 25 & $83.3 \%$ \\
\hline \multirow{2}{*}{ Mastoid pneumatization } & well pneumatized mastoid & 5 & $16.7 \%$ \\
\cline { 2 - 4 } & sclerosed mastoid & 26 & $86.7 \%$ \\
\hline \multirow{2}{*}{ Combined pathology } & $\begin{array}{l}\text { blocked aditus and sclerosed } \\
\text { mastoid }\end{array}$ & 2 & $13.3 \%$ \\
\hline \multirow{2}{*}{ Sclerotic patches } & no sclerotic patches & 27 & $6.7 \%$ \\
\cline { 2 - 4 } & Tympanosclerosis & 3 & $90.0 \%$ \\
\hline
\end{tabular}


Table (3): Correlation between CT and surgical findings: Middle ear space.

\begin{tabular}{|l|l|c|c|}
\hline \multicolumn{2}{|c|}{} & \multicolumn{2}{c|}{ Middle ear space (CT) } \\
\cline { 3 - 4 } & \multicolumn{2}{c|}{ Clear middle ear space } \\
\cline { 2 - 3 } Middle ear space (surgical) & Clear middle ear & 23 & $76.7 \%$ \\
\cline { 2 - 4 } & Polypoid middle ear mucosa & 3 & $10.0 \%$ \\
\cline { 2 - 4 } & Middle ear adhesions & 4 & $13.3 \%$ \\
\hline
\end{tabular}

Table (4): Correlation between CT and surgical findings: Ossicular chain.

\begin{tabular}{|l|l|c|c|c|c|}
\hline \multirow{2}{*}{\multicolumn{2}{|c|}{}} & \multicolumn{4}{c|}{ Ossicular chain (CT) } \\
\cline { 3 - 6 } & & \multicolumn{2}{c|}{ Intact ossicular chain } & \multicolumn{2}{c|}{ Disrupted } \\
\cline { 3 - 6 } \multicolumn{2}{|c|}{} & Count & $\%$ & Count & $\%$ \\
\hline \multirow{2}{*}{$\begin{array}{l}\text { Ossicular chain } \\
\text { (surgical) }\end{array}$} & Intact ossicular chain & 30 & $100.0 \%$ & 0 & $0.0 \%$ \\
\cline { 3 - 6 } & Disrupted & 0 & $0.0 \%$ & 0 & $0.0 \%$ \\
\hline
\end{tabular}

Table (5): Correlation between CT and Surgical findings: Aditus ad antrum

\begin{tabular}{|c|c|c|c|c|c|c|}
\hline & & \multicolumn{4}{|c|}{ Aditus ad antrum (CT) } & \multirow{3}{*}{ P value } \\
\hline & & \multicolumn{2}{|c|}{ Patent aditus } & \multicolumn{2}{|c|}{ Blocked aditus } & \\
\hline & & Count & $\%$ & Count & $\%$ & \\
\hline \multirow{2}{*}{$\begin{array}{l}\text { Aditus ad } \\
\text { antrum } \\
\text { (surgical) }\end{array}$} & Patent aditus & 24 & $96.0 \%$ & 1 & $20.0 \%$ & \multirow{2}{*}{0.001} \\
\hline & Blocked aditus & 1 & $4.0 \%$ & 4 & $80.0 \%$ & \\
\hline
\end{tabular}

Table (6): Correlation between CT and Surgical findings: Mastoid pneumatization.

\begin{tabular}{|c|c|c|c|c|c|c|}
\hline & & \multicolumn{4}{|c|}{ Mastoid pneumatization (CT) } & \multirow{3}{*}{$P$ value } \\
\hline & & \multicolumn{2}{|c|}{$\begin{array}{c}\text { Well pneumatized } \\
\text { mastoid }\end{array}$} & \multicolumn{2}{|c|}{ Sclerosed mastoid } & \\
\hline & & Count & $\%$ & Count & $\%$ & \\
\hline \multirow{2}{*}{$\begin{array}{c}\text { Mastoid } \\
\text { pneumatization } \\
\text { (surgical) }\end{array}$} & $\begin{array}{c}\text { Well pneumatized } \\
\text { mastoid }\end{array}$ & 26 & $100.0 \%$ & 0 & $0.0 \%$ & \multirow[t]{2}{*}{$<0.001$} \\
\hline & Sclerosed mastoid & 0 & $0.0 \%$ & 4 & $100.0 \%$ & \\
\hline
\end{tabular}

Table (7): Correlation between CT and Surgical findings: Sclerotic patches.

\begin{tabular}{|c|c|c|c|c|c|c|}
\hline \multirow{2}{*}{} & \multicolumn{4}{|c|}{ Sclerotic patches (CT) } & \multirow{2}{*}{ P value } \\
\cline { 3 - 6 } & & \multicolumn{2}{|c|}{$\begin{array}{c}\text { No sclerotic } \\
\text { patches }\end{array}$} & \multicolumn{2}{c|}{ Tympanosclerosis } & \\
\cline { 3 - 6 } & Count & $\%$ & Count & $\%$ & \\
\hline \multirow{2}{*}{$\begin{array}{c}\text { Sclerotic patches } \\
\text { (surgical) }\end{array}$} & $\begin{array}{c}\text { No sclerotic } \\
\text { patches }\end{array}$ & 27 & $100.0 \%$ & 0 & $0.0 \%$ & $<0.001$ \\
\cline { 2 - 6 } & Tympanosclerosis & 0 & $0.0 \%$ & 3 & $100.0 \%$ & \\
\hline
\end{tabular}

Table (8): Results of healing.

\begin{tabular}{|c|c|c|c|}
\hline \multicolumn{2}{|c|}{} & \multicolumn{2}{|c|}{} \\
\cline { 3 - 4 } & Healed & Count & $\%$ \\
\hline \multirow{2}{*}{ Success rate } & Failed & 28 & $93.3 \%$ \\
\cline { 2 - 4 } & & 2 & $6.7 \%$ \\
\hline
\end{tabular}




\section{DISCUSSION}

The purpose of myringoplasty is to repair a hole in the dermal membrane that improves patient hearing and reduces exposure to infection ${ }^{(13)}$.

Many factors contribute to the success or failure of surgery to correct the tympanic membrane perforation; these may be conveniently divided into mastoid and non mastoid factors. Mastoid factors include the extent of mastoid pneumatization and the presence of inflammatory disease in the mastoid. Non mastoid factors include general debility, technical error and Eustachian tube dysfunction ${ }^{(14)}$.

The role of Eustachian tube function in the successful outcome of myringoplasty remains controversial. Some studies have demonstrated a correlation between normal preoperative tubal function and successful grafting, but other studies have failed to confirm this ${ }^{(15)}$.

Some authors have argued that closure of tympanic membrane perforations and elimination of chronic drainage can be achieved effectively when performing tympanoplasty with or without mastoidectomy. They have been suggested further that mastoidectomy is not only unnecessary when treating chronic non-cholesteatomatous suppurative otitis media but also it increases patient risks and costs with little or no significant clinical outcome advantage ${ }^{(16)}$.

Cartilage has been successfully used in middle ear procedures, and has been shown to be well tolerated with minimal resorption over time. Cartilage is now used for a wide range of procedures. The main reason being the cartilage is easily available at the site of operation, is nontoxic, and causes less extrusion, minimum shrinkage and lateralization. Above all it is cost-effective. The hearing improvement within $15 \mathrm{~dB}$ has become almost a standard criterion for the analysis of surgical success. Reports in the literature suggest favorable anatomic results and low recurrence rates ${ }^{(\mathbf{1 7 )}}$.

CT scan has now largely replaced conventional polytomography for the imaging of the temporal bone and is capable of producing bone detail of outstanding quality. Potentially the most extensive use of temporal bone $\mathrm{CT}$ is in the evaluation of patients with chronic suppurative otitis media ${ }^{(\mathbf{1 8})}$.

CT scan added a new dimension to the study of middle ear diseases, middle ear masses and associated complications were visualized for the $1^{\text {st }}$ time ${ }^{(9)}$.
CT is very effective in evaluation of aditus blockage and residual tissue of the tympanomastoid region ${ }^{(12)}$.

Regarding middle ear spaces, the preoperative CT findings in this study showed that 30 cases with clear middle ear spaces $(100 \%)$ while, the surgical findings showed that 23 cases with clear middle ear spaces $(76.7 \%), 3$ cases with polypoid middle ear mucosa (10\%) and 4 cases with middle ear adhesions (13.3\%). Sudarshan and Juveria ${ }^{(19)}$ studied the CT observations on middle space and mucosa in cases of revision myringoplasty and compared it with surgical results using high resolution computed tomography (HRCT) of middle ear and temporal bone via Siemens 64 slice CT scanner. Axial, coronal and sagittal sections taken were of $1 \mathrm{~mm}$ thickness and $1 \mathrm{~mm}$ interval. The study was conducted on 25 patients with failed myringoplasty. The CT results of the study showed 22 patients with normal middle ear spaces with no soft tissue masses or granulations (88\%).Also, there were 3 patients with fibrous adhesions between lower part of ossicular chain and walls of mesotympanum (12\%).These CT findings showed accuracy $100 \%$ in comparison with surgical findings .The difference in results seems to be due to using of HRCT with small thin cuts.

Regarding ossicular chains, the preoperative CT findings in this study showed that 30 cases with intact ossicular chain (100\%) and the surgical findings also showed 30 cases with intact and mobile ossicular chain $(100 \%)$. This revealed that accuracy of CT scan in assessment of ossicles in cases of failed myringoplasty is $100 \%$. These results coincide with results of Kumaran et al. ${ }^{(18)}$ who studied the role of preoperative CT scan in surgical correlation in cases of recurrent CSOM. the study was conducted on 25 patients with recurrent CSOM from February 2006 to February 2008 at PSG institute of medical science and research. It revealed that accuracy of CT scan in ossicular assessment is 96\% (only one case showed erosion of incus while surgical assessment was free).

Regarding aditus ad antrum, the preoperative CT findings in this study showed that 25 cases with patent aditus ad antrum (83.3\%) and 5 patients with blocked aditus (16.7\%). The surgical findings was identical to CT findings showing 25 cases with patent aditus ad antrum (83.3\%) and 5 patients with blocked aditus (16.7\%).This revealed that accuracy of CT scan in assessment of aditus ad antrum in cases of failed myringoplasty is $100 \%$. These findings coincide with results of El-Kady et al. ${ }^{(20)}$ who studied the role of preoperative CT scan in assessment of aditus ad antrum patency in cases of failed myringoplasty with surgical 
Correlation Between Role of Preoperative Computerized Tomography...

correlation. The study was conducted on 40 patients with recurrent CSOM.CT scanning detected 29 cases with patent aditus ad anrtum $(72.5 \%)$ and 11 cases with blocked aditus $(27.5 \%)$. The CT findings coincided with the operative findings with accuracy in aditus ad antrum assessment $100 \%$.

Also our results coincide with results of Arvind et al. ${ }^{(21)}$ who analyzed the results of preoperative CT with surgical findings regarding aditus ad antrum patency in cases of revision myringoplasty. The study was conducted on 35 patients with recurrent CSOM. CT scanning detected 29 cases with patent aditus ad anrtum $(82.8 \%)$ and 6 cases with blocked aditus (17.2\%). The CT findings coincided with the operative findings with accuracy in aditus ad antrum assessment $100 \%$.

Regarding mastoid pneumatization, the preoperative CT findings in this study showed that 26 cases with well pneumatized mastoid (86.7\%) and 4 patients with sclerosed mastoid (13.3\%). The surgical findings was identical to CT findings showing 26 cases with well pneumatized mastoid (86.7\%) and 4 patients with sclerosed mastoid (13.3\%). This revealed that accuracy of CT scan in assessment of mastoid pneumatization in cases of failed myringoplasty is $100 \%$.

These results coincide with results of Ginni $\boldsymbol{e t}$ al. ${ }^{(22)}$ who studied the role of preoperative CT scan of mastoid air system with surgical correlation in cases of recurrent CSOM. The study was conducted on 25 patients with failed myringoplasty in the Department of Otorhinolaryngology and Radiodiagnosis at Shimla. The study showed 24 patients with pneumatized mastoid (96\%) and only one patient with sclerosed mastoid (4\%) with CT findings correlated very well to surgical findings $(100 \%)$ in all patients of revision myringoplasty.

Regarding presence of sclerotic patches, CT findings revealed 27 patients with no sclerotic patches (90\%) and 3 patients with sclerotic patches limited to ear drum (10\%). The surgical findings was identical to CT findings showing 27 cases with no sclerotic patches $(90 \%)$ and 3 patients with sclerotic patches ie :tympanosclerosis (10\%). This revealed that accuracy of CT scan in detection of sclerotic patches is $100 \%$. Our results coincide with results of Boyraz et al. (23) who analyzed the importance of computed tomography examination of temporal bone in detecting tympanosclerosis in failed myringoplasty. The study was conducted on 19 patients with recurrent CSOM between January 2006 and May 2006 at the Department of Otolaryngology, Head and Neck Surgery, Izmir Atatürk Training and Research Hospital. CT scanning detected 16 cases with no sclerotic patches $(84.2 \%), 2$ cases with tympanosclerotic patches $(10.5 \%)$ and 1 case with sclerotic patch in the middle ear $(5.3 \%)$. The CT findings coincided with the surgical findings with accuracy $100 \%$.

The outcome of our cases was successful healing of tympanic membrane in 28 cases $(93.3 \%)$ and 2 cases with residual perforation $(6.7 \%)$ due to postoperative infection. These results coincide with results of Dornhoffer ${ }^{(24)}$ who reported 96 patients with recurrent CSOM were operated using cartilage graft to close tympanic membrane perforation. Tympanic membrane closure was achieved in about $95 \%$ of patients.

This study proved that CT is very effective in evaluation of ossicular chain, aditus ad antrum and mastoid pneumatization and not very effective in evaluation of middle ear spaces in cases of failed myringoplasty. Multi slice CT scan with $1 \mathrm{~mm}$ thickness and $1 \mathrm{~mm}$ interval may be helpful in assessment of middle ear cavity.

Also CT scan helps in detection of causes of failure of myringoplasty and plays a role in choice of line of treatment in cases of recurrent CSOM.

\section{CONCLUSION}

Comparing these results with those of other researches using similar techniques, revealed that they are almost similar regarding the accuracy of CT scanning and success rate.

\section{REFERENCES}

1. Merchant SN (2005): Ossiculoplasty and tympanoplasty in chronic otitis media. In: NadolJB, McKenna MJ, editors. Surgery of the Ear and Temporal Bone. $2^{\text {nd }}$ ed. USA:Lippincott Williams and Wilkins, pp.305-325.

2. Bhat KV, Naseeruddin K, Nagalotimath US et al. (2008): Cortical mastoidectomy in quiescent, tubotympanic, chronic otitis media: Is it routinely necessary? J Laryngol Otol., 10:1-8.

3. Sheehy JL (1980): Myringoplasty: a review of 472 casers. Ann otol., 89: 331-335.

4. Jones RO (2004): Myringoplasty. In: Haberman RS, Middle Ear and Mastoid Surgery. https://www.scribd.com/.../Middle-Ear-and-MastoidSurgery-R-Haberman-Thieme-2004

5. Merchant SN, Rosowski JJ, Shelton C (2009): Reconstruction of the middle ear. In: Snow JB, Wackym PA, Ballenger JJ, editors. Ballenger' sotorhinolaryngologyhead and neck surgery.United States: People's Medical Publishing House, pp.239-45.

6. Ars B and Ars-Piret N (1994): Middle ear pressure balance under normal conditions. Specific role of the middle ear structure. ActaOtorhinolaryngol Belg., 48:339-42.

7. Uzun C, Velepic M, Manestar D et al. (2003): Cartilage Palisade Tympanoplasty, Diving and 
Eustachian Tube Function. Otology \& Neurotology, 350:1.

8. Kartush JM (2002): Tympanic membrane patcher: A new device to close tympanic membrane perforation in an office setting, Am. J. Otol., 21:615.

9. Swartz JD and Harnsberger HR (1998): The middle ear and mastoid imaging of the temporal bone, third edition. Thieme, New York, pp 85-107 3.

10. Johnson DW, Voorhees RL, Lufkin RB et al. (1983): Cholesteatomas of the temporal bone: role of computed tomography radiology, 148: 733-737.

11. Casselman JW, Kuhweide R, Deimling M (1996): Constructive interference in steady state-3DFT MR imaging of the inner ear and cerebellopontine angle. AJNR., 14:47-57.

12. Gorur C and Umittales D (2006): The computed tomographical and tympanometrical evaluation of mastoid pneumatization and attic blockage in patients with chronic otitis media with effusion. International journal of pediatric otorhinolaryngology, 70 (3): 481485.

13. Sarkar S, Roychoudhury A, Roychoudhury K (2009): Tympanoplasty in children. Eur Arch Otorhinolaryngol., 266:627-33.

14. Uyar Y, Keles B, Koc S et al. (2006): Tympanoplasty in pediatric patients.Int J Pediatr Otorhinolaryngol., 70(10):1805-1809.

15. Todd NW (2000): There is no accurate tests of Eustachian tube function, Arch Otolaryngol Head and Neck Surgery, 126: 1041-1042.

16. Usami S, Iijima N, Fujita $S$ et al. (2001): Endoscopicassisted myringoplasty. ORL J OtorhinolaryngolRelat Spec., 63: 287-90.

17. Iacovou E, Vlastarakos P, Kyrodimos E et al. (2013): Is cartilage better than temporalis muscle fascia in type I tympanoplasty? Implications for current surgical practice; Eur Arch Otorhinolaryngol, DOI 10.1007/s00405-012-2329-4.

18. Kumaran A, Colbert R, Vintha $G$ et al. (2014): Computer tomography and surgical correlation in unsafe ear. IOSR Journal of Dental and Medical Sciences , 13(6): 7-12.

19. Sudarshan $R$ and Juveria $M$ (2016): Role of CT Mastoids in the Diagnosis and Surgical Management of Chronic Inflammatory Ear Disease. https://medwinpublishers.com/OOAJ/OOAJ16000144.p $\mathrm{hp}$ ?id=8

20. El-kady AS, Haroun Y, Kassem MK et al. (2009): The value of computed tomography scanning in assessment of aditus ad antrum patency and choice of treatment line in revision myringoplasty. Med. J. Cairo Univ., 77(2): 53-57.

21. Arvind V, Maithani T, Kumar A et al. (2015): Clinical and Radiological predictability of aditus patency in mucosal COM with sclerosed mastoid: An analytical study.Otorhinolaryngology Clinics: An international journal, 7(3):121-124.

22. Ginni D, Mohan C, Mahajan M et al. (2014): Correlation of preoperative HRCT findings with surgical findings in Unsafe CSOM. Journal of Dental and Medical Sciences , 13(1):120-125.

23. Boyraz E, Erdogan N, Kazikdas C et al. (2009): The importance of computed tomography examination of temporal bone in detecting tympanosclerosis. Journal of Ear, Nose, and Throat, 19(6):294-298.

24. Dornhoffer JL (2006): Cartilage tympanoplasty: Indications, techniques, and outcomes in a 1000- patient series. Laryngoscope, 113:1844-1856. 